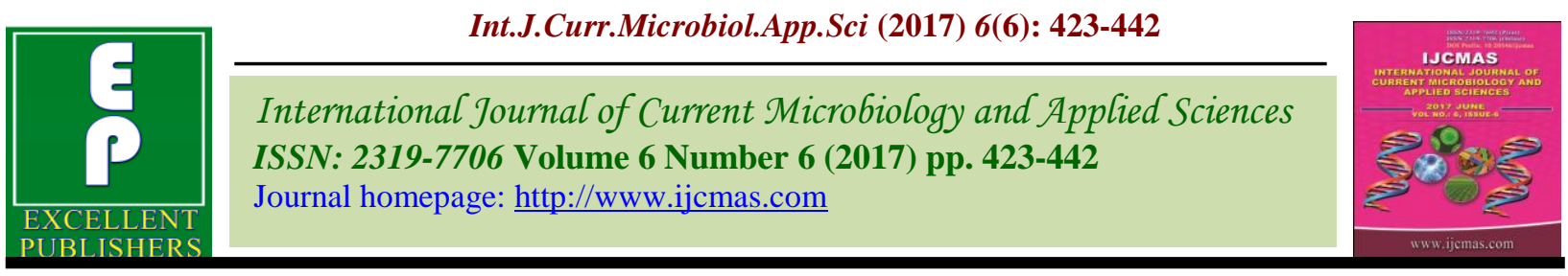

Original Research Article

https://doi.org/10.20546/ijcmas.2017.606.050

\title{
Aerobic Bacterial Isolates and their Antibiotic Susceptibility Pattern from Pus Samples in a Tertiary Care Government Hospital in Tamilnadu, India
}

\author{
Sukumar Nirmala ${ }^{1 *}$ and Rajesh Sengodan ${ }^{2}$ \\ Department of Microbiology, Government Mohan Kumaramangalam \\ Medical College and Hospital, Salem, Tamil Nadu, India \\ *Corresponding author
}

\begin{abstract}
A B S T R A C T
This prospective study was conducted in our tertiary care set up to detect the bacterial isolates with antibiotic susceptibility pattern from pus samples, along with

Keywords

ESBL, MRSA,

Bacterial pathogens isolated,

Antibiotic

susceptibility

pattern.

Article Info

Accepted:

04 May 2017

Available Online:

10 June 2017 sociodemographic factors leading to wound infections and mainly to provide relevant data of antibiotics preferred for each bacterial isolate. This would be a baseline for formulation of necessary policies for antibiotic use in our set up. In our study almost $1836(75 \%)$ of pus samples showed bacterial growth. 1303 (71\%) male patients were infected whereas only 533(29\%) female patients were infected. Common age group involved is 21-40 years. Gram negative bacteria were isolated more than gram positive bacteria. Out of which Klebsiella species was the most predominant bacterial pathogen 635(35\%) followed by Staphylococcus aureus 334 (18\%), Escherichia coli 253 (14\%), Coagulase negative Staphylococcus species 204 (11\%), Pseudomonas aeruginosa 153 (8\%), Proteus species 126 (7\%), Citrobacter species 51 (3\%), Enterococcus species 43(2\%) and Acinetobacter species 37 (2\%). Highest incidence was due to ESBL isolates of Pseudomona aeruginosa $90(59 \%)$ followed by Klebsiella sp 312(49\%), E. coli 124 (49\%), Proteus sp. 62(49\%), Acinetobacter sp 18 (48\%) and Citrobacter sp 18 (35\%).MRSA isolates were 96(43\%) in Staphylococcus aureus isolates followed by $84(41 \%)$ in Coagulase negative Staphylococcal species.
\end{abstract}

\section{Introduction}

Skin acts as a barrier and prevents the entry of microbes into our body unless the mechanism is breached due to any injury, trauma or surgical intervention. Thus wounds formed get infected by bacterial pathogens (Rugira Trojan, 2016 and Aniruddha, et al., 2015). Despite their starting point, all injuries are due to the microorganisms present inside or outside the human body or both (Maria Ayub et al., 2015).

Wound infections have become a global problem in surgery due to long hospital stay,

high treatment expenditure and emergence of multidrug resistant bacteria leading to gross mutilation of patients affected (Rugira Trojan, 2016). Wound infections have become the most important cause for morbidity and mortality (Ezekiel Olugbenga Akinkunmi et al., 2014) the overall incidence of wound sepsis in India is $10-33 \%$ (Akimoto et al., 1994).

Due to the multi diversity of infection causing bacteria this study was conducted to provide relevant data which would be of immense 
help to serve the patients attending our tertiary care government hospital. In spite of the progress in surgical techniques and antibiotic prophylaxis, wound infections remain the commonest postoperative complications and one of the most frequently encountered nosocomial infections worldwide (Nichols et al., 2004). These lead to increasing demand for advanced surgical techniques like laparoscopy to decrease significant morbidity.

As a result of these problems, routine surveillance for hospital acquired wound infections, including wound infections, operation theatres, and critical care units is recommended by the CDC (Oluwatosin, 2005). This has become most important part of infection control policy.

Wound infections that occur postoperatively also depend on the type of surgery which is classified as clean, clean contaminated, contaminated and dirty (Lilani et al., 2005). Wound infections are severe in case of contaminated and dirty surgical procedures where there is a severe breach in aseptic surgical techniques adopted due to surgery (Ezekiel Olugbenga Akinkunmi et al., 2014).

The investigation of the microbiologic spectrum and antibiotic susceptibility of isolates from pus samples is therefore of increasing importance bearing in mind the increasing antibiotic resistance by microorganisms and the high incidence of surgical infections caused by these resistant organisms (Dryden et al., 2005). It has been documented that the type of organisms infecting surgical wound is a reflection of the body system involved in the surgical operation. According to the reports, these organisms which are normal inhabitants of the body system usually become opportunistic pathogens when their niche is violated. Selection of an effective antimicrobial agent for a microbial infection requires knowledge of the potential microbial pathogen, an understanding of the pathophysiology of the infectious process and an understanding of the pharmacology and pharmaco kinetics of the intended therapeutic agents (Kelwin et al.,, 1999). Both aerobic and anaerobic bacteria have been implicated in wound infections which commonly occur under hospital environment (Cerceo et al., 2016).

Rapid emergence of multidrug-resistant bacteria poses a serious threat to public health globally due to the limited treatment options and luke warm discovery of new classes of antibiotics. The incidence of MRSA (Methicillin Resistant Staphylococcus aureus) in India ranges from 30 to $70 \%$ (Subrata Roy, 2016).

Although Staphylococcus aureus is a commensal of humans, it is also a frequent cause of human infections which may become serious if caused by antimicrobial resistant strains. Antibiotic resistant $S$. aureus, especially MRSA, are equally adapted to hospitals and outer environments evolving as major pathogens of public health concern. ESBLs were found in 63.6 per cent, 66.7 per cent of $E$. coli and $K$. pneumoniae isolates (Ankur Goyal et al., 2009).

Incidence of extended spectrum beta lactamase producing Escherichia coli among patients, healthy individuals and in the environment is $60 \%, 38 \%$ and $10 \%$ respectively (George et al., 2014). ESBL producing strains are Klebsiella pneumoniae, Klebsiella oxytoca and Escherichia coli (Sanders et al., 1996). The situation is serious in developing countries due to irrational prescriptions of antimicrobial agents.

The inoculum size, virulence and invasive capability of the organisms have been reported to influence the risk of infection. 
Moreover, the physiological state of the tissue in the wound and immunological integrity of the host also has equal importance in determining occurrence of infection (Mengesha et al., 2014).

The emergence of drug-resistant organisms in both hospitals and the community is a major concern. Surveillance studies have provided important information about changes in the spectrum of microbial pathogens and trends in the antimicrobial resistance patterns in nosocomial and community-acquired infections and continued monitoring of antimicrobial resistance patterns in hospitals is essential to guide effective empirical therapy (BN Chaudhuri et al., 2007).

Focusing on this ever increasing issue of multidrug resistance due to inappropriate use of antibiotics and considering the fact that no data was available on ESBL producing GNB's and methicillin resistant Staphylococcus aureus in our region, the current study was undertaken in our department to provide adequate and relevant data for proper selection and use of antibiotics.

Anaerobic bacteria also form an equal part in causing wound infections. As there were no proper resources for culture of anaerobic bacteria in our facility it was not done.

The main objective of this study includes, to isolate the aerobic pyogenic bacteria from pus samples; to determine the antibiotic susceptibility pattern of gram negative and gram positive bacteria isolated from pus samples; to determine the socio demographic factors involved in this study; to provide relevant information to the clinicians of our hospital regarding the antibiotic to be prescribed to decrease the emergence and spread of multidrug resistant bacteria.

\section{Materials and Methods}

\section{Study area}

This prospective study was conducted in Diagnostic Microbiology Laboratory, Government Mohan Kumaramanalam Medical College Hospital, Salem, a tertiary care hospital situated in the centre of the city with 1971 beds and cateringa minimum of 4500 patients per day from Dharmapuri, Namakkal, Krishnagiri, Hosur and districts around Salem.

\section{Study design}

This study included all the pus samples caused by wound infections received in Microbiology laboratory for bacterial culture and antibiotic susceptibility testing from both in patients and outpatients irrespective of age and sex from all the departments of this hospital.

\section{Acceptance criteria}

All the pus samples collected aseptically by sterile aspiration in sterile containers and in sterile swabs were accepted for this study

\section{Rejection criteria}

Pus samples received in unsterile containers and with containers soaked with pus due to improper closure of screw caps were rejected.

\section{Study period}

This prospective study was conducted for a period of six months from July 2016 till December 2016 in the Department of Diagnostic Microbiology in accordance with standard protocols and ethical guidelines. Direct Microscopic examination of gram stained pus smears was done. Pus cells and bacteria present were noted as per protocol. 
Bacterial culture was performed by inoculation of pus aspecticallyonto nutrient agar, 5\% sheep blood agar and Mac Conkey agar plates and incubated aerobically at 35$37^{\circ} \mathrm{C}$ for $24-48$ hours. Bacterial colonies were identified and characterised based on gram staining, morphology of colonies, and biochemical reactions using standard microbiological methods (Koneman, 2006).

Bacterial suspensions were prepared for each bacterial isolate separately and turbidity matched with 0.5 McFarland Standard and lawn culture of the bacteria was performed on surface of Mueller Hinton agar plates.

Antibiotics discs containing Ampicillin (Amp) - $10 \mu \mathrm{g}$, Piperacillin (Pi) - $100 \mu \mathrm{g}$, Cefazolin $(\mathrm{Cz})$ - $30 \mu \mathrm{g}$, Cephalexin $(\mathrm{Cn})-30$ $\mu \mathrm{g}$, Cefuroxime $(\mathrm{Cxm})-30 \mu \mathrm{g}$, Cefixime (Cfm) -5 $\mu \mathrm{g}$, Ceftriaxone (Ctr) $-30 \mu \mathrm{g}$, Ceftazidime (Caz) -30 $\mu$ g, Cefotaxime (Ctx) $30 \mu \mathrm{g}$, Azithromycin (Azm) - $15 \mu \mathrm{g}$, Clarithromycin (Clr) -15 $\mu \mathrm{g}$, Doxycycline (Do) -30 $\mu \mathrm{g}$, Penicillin G- 100 units, Amikacin (AK) - $30 \mu \mathrm{g}$, Gentamicin(Gen) -10 $\mu \mathrm{g}, \quad$ Tobramycin (Tob) $\quad-10 \mu \mathrm{g}$, Netilmicin(Net) $-10 \mu \mathrm{g}$, Cotrimoxazole (Cot) -1.25/23.75 $\mu \mathrm{g}$, Ciprofloxacin(Cip) -5 $\mu \mathrm{g}$, Levofloxacin (Le) $-5 \mu \mathrm{g}$, Gemifloxacin(Gem) -5 $\mu \mathrm{g}, \quad$ Imipenem (Ipm) $-10 \mu \mathrm{g}$, Cefaperazonesulbactum (Cfs) - 75/10 $\mu \mathrm{g}$, Amoxycillinclavulanic acid (Amc) -20/10, Piperacillintazobactum (Pit) - 100/10 $\mu \mathrm{g}$, Ceftriaxone / clavulanic acid (Cac) -30 /10 $\mu \mathrm{g}$, Ceftazidime / clavulanic acid (Cfc) -30 $/ 10 \mu \mathrm{g}$, Vancomycin $-30 \mu \mathrm{g}$ were obtained from Himedia Laboratories and used as per manufacturer's instructions.

High level gentamicin disc for used exclusively for testing Enterococcus species. Antibiotic susceptibility test for all bacterial isolates were performed based on Modified Kirby Bauer's disc diffusion method. Antibiotic discs were placed on the agar plates and incubated overnight at $37^{\circ} \mathrm{C}$ for 24hours. Thezones of inhibition were measured and the isolates were classified as susceptible, intermediate, and resistant according to CLSI guidelines (CLSI, 2017). Quality control strains used for AST were Escherichia coli ATCC 25922, Pseudomonas aeruginosa ATCC 27853 and Klebsiella pneumoniae ATCC 700603.

Drug resistant gram negative bacilli producing extended spectrum beta lactamases (ESBL) and Methicillin resistance in Staphylococcal isolates (MRSA) were identified as per the methods of detection given below

\section{ESBL screening test}

As per the CLSI Screening criteria, the strain was considered to be "suspicious" of ESBL production if a zone diameter for the third generation cephalosporins was recorded as $<=$ $22 \mathrm{~mm}$ for ceftazidime, and $<=25 \mathrm{~mm}$ for ceftriaxone. For E. coli and Klebsiella strains ceftriaxone zone $<=25 \mathrm{~mm}$ and ceftazidime zone diameter of $<=22 \mathrm{~mm}$ is considered to be resistant and indicates ESBL production.

\section{ESBL Confirmatory Method}

All the strains which were screened out for ESBL production were also subjected to confirmation by using the Phenotypic confirmatory disc diffusion test (CDDT), as recommended by the CLSI.

Discs of ceftazidime+ clavulanic acid (Cfc) $30 \mu \mathrm{g} / 10 \mu \mathrm{g}$,andCeftriaxone / clavulanic acid (Cac)-30 $\mu \mathrm{g} / 10 \mu \mathrm{g}$, (Hi - media) were used as per CLSI guidelines. Organism was considered as ESBL producer if there was a more than or equal to $5 \mathrm{~mm}$ increase in zone diameter for ceftazidime and ceftriaxone tested in combination with clavulanic acid versus its zone when tested alone. 


\section{Detection of MRSA}

Methicillin resistant Staphylococcus aureus strains were identified based on susceptibility to Cefoxitin $30 \mu \mathrm{g}$ disc which is considered as surrogate marker for oxacillinused for detection of methicillin resistant isolates as per CLSI guidelines

\section{Cefoxitin disc diffusion method}

All strains were tested with $30 \mu \mathrm{g}$ cefoxitin(Cx) discs (Hi-Media) on MuellerHinton agar plates. For each strain, a bacterial suspension adjusted to $0.5 \mathrm{McF}$ arland was used. The zone of inhibition was determined after $16-18$ hour incubation at $35^{\circ} \mathrm{C}$. Zone size was interpreted according to CLSI criteria: susceptible, $>=22 \mathrm{~mm}$; resistant, $<=21 \mathrm{~mm}$. Those strains exhibiting zone diameter of $<=21 \mathrm{~mm}$ were considered as methicillin resistant.

Age, sex, clinical details of the patients included in this study were recorded in a proforma after obtaining informed consent from all the patients included in the study.

\section{Results and Discussion}

Total number of pus samples included in this study was 2455. Out of these 1836 (75\%) samples showed growth of bacterial colonies and $619(25 \%)$ samples did not show bacterial growth even after 48 hours of aerobic incubation as indicated in figure 1 .

\section{Socio demographic factors in patients with wound infections}

In our study almost $1836(75 \%)$ of pus samples showed bacterial growth. 1303(71\%) male patients were infected whereas only $533(29 \%)$ female patients were infected among the pus samples received. Common age group involved is $21-40$ years.
Among 532 (22\%) samples received from Accident and Emergency trauma ward, 459 (25\%) of samples showed bacterial growth followed by 422 (23\%), 294 (16\%) and 257 (14\%) from burns ward, postoperative ward, diabetology ward respectively as described in table 1 .

\section{Bacteria isolated were as follows}

Klebsiella species was the most predominant bacterial pathogen 635 (35\%) followed by Staphylococcus aureus 334 (18\%), Escherichia coli 253 (14\%), Coagulase negative Staphylococcus species 204 (11\%), Pseudomonas aeruginosa 153 (8\%), Proteus species 126 (7\%), Citrobacter species 51 (3\%), Enterococcus species, 43(2\%) and Acinetobacter species 37 (2\%) as described in figure 3.

Polymicrobial infection with two different organisms occurred in $22 \%$ of cases. Combined organisms were Pseudomonas aeruginosa with Staphylococcus aureus in $15 \%$ followed by Klebsiella species with Coagulase negative Staphylococcus species in $7 \%$ of isolates.

\section{Extended spectrum beta lactamase (ESBL)} producing gram negative bacterial isolates

ESBL producing isolates were found to be highest in Klebsiella species 311 (49\%), Escherichia coli124 (49\%) followed by Proteus spesies 62 (49\%), Acinetobacter species $18(48 \%)$ and Pseudomonas aeruginosa 90 (59\%). All the ESBL producing bacterial isolates were $100 \%$ sensitive to carbapenem group of drugs. Hence Amp C metallobeta lactamase detection was not done. Screening test using Ceftriaxone, Ceftazidime disc was done and confirmation of the same was also done CDDT using Ceftriaxone clavulanic acid and 
Ceftazidime clavulanic acid antibiotic disc as per guidelines.

ESBL production was detected in all the gram negative bacilli in varying percentage. Total gram negative bacilli producing ESBL was $626(50 \%)$. Highest incidence was due to ESBL isolates of Pseudomonas aeruginosa 90 (59\%) followed by Klebsiella sp 312(49\%), E. coli 124 (49\%), Proteus sp. 62(49\%), Acinetobacter sp 18 (48\%) and Citrobacter sp $18(35 \%)$ as described in table 2 and figure 4.

\section{Methicillin resistant Staphylococcal isolates}

Methicillin resistance was detected by Cefoxitin disc diffusion method.

MRSA isolates were 96(43\%) in Staphylococcus aureus isolates followed by $84(41 \%)$ in Coagulase negative Staphylococcal species. All the gram positive bacteria isolated were found to be sensitive to Vancomycin (100\%). No vancomycin resistant strain was detected including Enterococcus species.

Klebsiella species isolated were $64 \%$ sensitive to piperacillin tazobactum, $63 \%$ sensitive to amikacin, $61 \%$ sensitive to tobramycin, $52 \%$ sensitive to ceftazidime, $51 \%$ to ceftriaxone, $49 \%$ sensitive to cefaperazone tazobactum and gemifloxacin, $48 \%$ sensitive to ceftriaxone.

Antibiotic resistance of Klebsiella species was higher to oral first, second and third generation cephalosporins and other beta lactam group of antibiotics.

E. coli isolates exhibited antibiotic susceptibility of $100 \%$ to imipenem, followed by $75 \%$ to gemifloxacin, $70 \%$ to amikacin, $63 \%$ to tobramycin, $63 \%$ to ciprofloxacin and $49 \%$ sensitivity to cefaperazone sulbactum combination.
Pseudomonas aeruginosa is $100 \%$ sensitive to Imipenem, $85 \%$ to tobramycin, $85 \%$ to Ciprofloxacin, $71 \%$ to netilmicin, followed by Piperacillin (51\%), Ceftazidime (58\%), cefaperazone sulbactum (59\%), and piperacillin tazobactum $(58 \%)$

Gram positive bacteria mainly $S$. aureus exhibited $100 \%$ sensitivity to vancomycin and linezolid followed by $60 \%$ sensitivity to all beta lactam antibiotics and $59 \%$ to aminoglycosides.

All the ESBL producing gram negative bacilli isolated in our study showed $100 \%$ susceptibility to Carbapenems. All MRSA isolated showed $100 \%$ susceptibility to Vancomycin and Linezolid.

In our study almost $1836(75 \%)$ of pus samples showed bacterial growth. Male patients were more infected than female patients. Common age group involved is $21-$ 40 years. This was similar to the study by Vijeta Sharma et al., (2015).

Gram positive bacteria were isolated from $581(32 \%)$ pus samples and gram negative bacteria were isolated from 1255 (68\%) pus samples. Mixed growth was seen in $22 \%$ samples and no growth in $25 \%$ of pus samples.

Gram-negative bacteria were the dominant isolates from pus samples compared to Grampositive bacteria which are in agreement to several earlier studies. This was similar to the studies by Rugira Trojan et al., (2016), Bhatt and Vassikar (2010), Rajeshwar Rao et al., (2014), Sarathbabu et al., (2012), Kritu Panta et al.,, (2013), Ravichitra (2014), Rajan (2014), Sankarankutty et al., (2014) and Aizza Zafar (2008).

Bacterial pathogens were isolated from patients with the isolation rate of $75 \%$ similar to study by Dagnachew Muluye et al., (2014) 
This was higher than the results reported Mulu et al., (2006) and Hima Bindu Mantravadi et al., (2015).

Most infected wounds were due to trauma or injury, followed by postoperative infection of surgical wounds, diabetic foot ulcers followed by other minor lesions similar to the study by Ashby et al., (2010).

Polymicrobial infection with two different organisms occurred in $22 \%$ of cases. Combined organisms were Pseudomonas aeruginosa with Staphylococcus aureus in 15\% followed by Klebsiella species with Coagulase negative Staphylococcus species in $7 \%$ of isolates.

As per the study conducted by Lucinda $\mathrm{J}$ Bessa et al., (2013), polymicrobial infection was found in $27 \%$ of the samples and was mainly constituted with two species. The most common association was $S$. aureus/P. aeruginosa. Similar to the association among organisms isolated in our study.

Klebsiella species was the most predominant organism followed by Staphylococcus aureus, Escherichia coli, Pseudomonas aeruginosa, Acinetobacter species similar to study by Vijeta Sharma et al., (2015).

Most ESBL producers were isolated in Klebsiella pneumonia and Escherichia coli and Pseudomonas aeruginosa. $43 \%$ of Staphylococcus species was methicillin resistant and all the isolates were sensitive to Vancomycin.

In this study highest occurrence were observed in male $\mathrm{n} \%=1303(71 \%)$ as compared to female $\mathrm{n}(\%)=533(29 \%)$. It was correlated with Siddqui et al., (2002) study which shows male preponderance $(72 \%)$. Most commonly affected age group ranges between $21-40$ years. This was similar to studies by Bhatt and Vassikar (2010) and Rajeshwar Rao et al., (2014).

Klebsiella sp. was the most predominant organism $635(35 \%)$. This study was similar to other authors like Sarathbabu et al., (2012), Rajeshwar Rao et al., (2014), Kritu Panta et al., (2013) and Ravichitra (2014), Ananth and Rajan (2014) and Sankarankutty et al., (2014).

Staphylococcus aureus was the next common organism $334(8 \%)$ in the present study and correlated with other workers like Haitham et al., (2011), Iman Hussain et al., (2012). Aizza Zafar (2008) also has shown as second most disease causing organism.

However many workers have found most predominant organism in their studies like Farzana et al., (2013), Ehmer Al-Ibran (2013) and Soumya Kaup and Jaya Sankarankutty (2014).

The present study reveals the incidence of infections due to Klebsiella species and their tendency towards antibiotic resistance. Multidrug resistant bacteria are emerging worldwide which causes major public health problems and challenges to health care.

Klebsiella species are $100 \%$ sensitive to Imipenem, $63 \%$ of Amikacin,52\% Ceftazidime, $49 \%$ Gemifloxacin (which is considered as most preferred drug for treatment of Klebsiella species), $48 \%$ Cefotaxime,, $46 \%$ Ciprofloxacin, $22 \%$ Tetracycline and 20\% Amoxycillinclavulanic acid. 
Table.1 Socio demographic factors in patients with wound infections

\begin{tabular}{|l|l|l|l|}
\hline Demographic factors & Infected No. (\%) & Not infected No.(\%) & Total samples \\
\hline Male & $1303(71 \%)$ & $611(99 \%)$ & $1914(78 \%)$ \\
\hline Female & $533(29 \%)$ & $8(1 \%)$ & $541(22 \%)$ \\
\hline Total & 1836 & 619 & 2455 \\
\hline Age in years & $184(10 \%)$ & $39(6 \%)$ & $223(9 \%)$ \\
\hline $10-20$ & $587(32 \%)$ & $69(11 \%)$ & $656(27 \%)$ \\
\hline $21-30$ & $454(25 \%)$ & $39(6 \%)$ & $493(20 \%)$ \\
\hline $31-40$ & $220(12 \%)$ & $273(44 \%)$ & $493(20 \%)$ \\
\hline $41-50$ & $204(11 \%)$ & $190(32 \%)$ & $394(16 \%)$ \\
\hline $51-60$ & $187(10 \%)$ & $9(1 \%)$ & $196(8 \%)$ \\
\hline $61-70$ & 1836 & 619 & 2455 \\
\hline Total & $459(25 \%)$ & $81(13 \%)$ & $540(22 \%)$ \\
\hline Wards and lesions & $422(23 \%)$ & $94(15 \%)$ & $516(21 \%)$ \\
\hline Trauma ward & $294(16 \%)$ & $123(20 \%)$ & $417(17 \%)$ \\
\hline Burns ward & $257(14 \%)$ & $111(18 \%)$ & $368(15 \%)$ \\
\hline Postoperative wards & $142(23 \%)$ & $344(14 \%)$ \\
\hline Diabetic foot Ulcers & $202(11 \%)$ & $68(11 \%)$ & $270(11 \%)$ \\
\hline Abrasions, ulcers & $202(11 \%)$ & 619 & 2455 \\
\hline Others & 1836 & $580(94 \%)$ & $2371(97 \%)$ \\
\hline Total & $1791(98 \%)$ & $39(6 \%)$ & $84(3 \%)$ \\
\hline Patients & $45(2 \%)$ & 619 & 2455 \\
\hline Inpatients & 1836 & \\
\hline Outpatients &
\end{tabular}

Table.2 Gram negative bacilli producing ESBL

\begin{tabular}{|l|l|l|l|}
\hline Bacteria isolated & ESBL isolates n (\%) & Non ESBL isolatesn(\%) & Total \\
\hline Klebsiella sp. & $312(49)$ & $323(51)$ & 635 \\
\hline Escherichia coli & $124(49)$ & $129(51)$ & 253 \\
\hline Proteus $\boldsymbol{s}$. & $62(49)$ & $64(51)$ & 126 \\
\hline Citrobacter $\boldsymbol{s p}$ & $18(35)$ & $33(65)$ & 51 \\
\hline Pseudomonas aeruginosa & $90(59)$ & $63(41)$ & 153 \\
\hline Acinetobacter $\boldsymbol{s p}$ & $18(48)$ & $19(52)$ & 37 \\
\hline Total & $624(50)$ & $629(50)$ & 1255 \\
\hline
\end{tabular}


Table.3 Antibiotic susceptibility of gram negative bacteria to various groups of antibiotics

\begin{tabular}{|c|c|c|c|c|c|c|c|c|c|c|c|c|}
\hline \multirow{3}{*}{$\begin{array}{l}\text { Organism } \\
\text { n (\%) } \\
\text { Ant ibiotics }\end{array}$} & \multicolumn{2}{|c|}{ Klebsiella $\$ p$} & \multicolumn{2}{|l|}{ Ecoli } & \multicolumn{2}{|c|}{ Pseudomonas sp. } & \multicolumn{2}{|l|}{ Proteus sp } & \multicolumn{2}{|c|}{ Citrobacter sp } & \multicolumn{2}{|c|}{ Acinetobacter $s p$} \\
\hline & \multicolumn{2}{|l|}{$635(35)$} & \multicolumn{2}{|l|}{$253(41)$} & \multicolumn{2}{|l|}{$153(8)$} & \multicolumn{2}{|l|}{$126(7)$} & \multicolumn{2}{|l|}{$51(3)$} & \multicolumn{2}{|l|}{$37(2)$} \\
\hline & $\mathbf{S}$ & $\mathbf{R}$ & $\mathbf{S}$ & $\mathbf{R}$ & $\mathbf{S}$ & $\mathbf{R}$ & $\mathrm{s}$ & $\mathbf{R}$ & $\mathbf{S}$ & $\mathbf{R}$ & $\mathbf{s}$ & $\mathbf{R}$ \\
\hline AK & $400(63)$ & $235(37)$ & $177(70)$ & $76(30)$ & $99(65)$ & $54(35)$ & $55(44)$ & $71(56)$ & $45(88)$ & $6(12)$ & $34(92)$ & $3(8)$ \\
\hline Gen & $362(57)$ & $273(43)$ & $146(58)$ & $107(42)$ & $66(43)$ & $87(57)$ & $35(28)$ & $91(72)$ & $43(85)$ & $8(15)$ & $27(72)$ & $10(28)$ \\
\hline Tob & $387(61)$ & $248(39)$ & $159(63)$ & $94(37)$ & $130(85)$ & $23(15)$ & $52(41)$ & $74(59)$ & $48(94)$ & $3(6)$ & $34(92)$ & $3(8)$ \\
\hline Net & $375(59)$ & $260(41)$ & $167(66)$ & $86(34)$ & $109(71)$ & $44(29)$ & $53(42)$ & $73(58)$ & $46(97)$ & $5(3)$ & $31(84)$ & $6(16)$ \\
\hline Amp & $133(21)$ & $502(79)$ & $116(46)$ & $137(54)$ & $37(24)$ & $116(76)$ & $60(48)$ & $66(52)$ & $15(29)$ & $36(71)$ & $10(28)$ & $27(72)$ \\
\hline $\mathrm{Pi}$ & $133(21)$ & $502(79)$ & $124(49)$ & $129(51)$ & $78(51)$ & $75(49)$ & $68(54)$ & $58(46)$ & $21(41)$ & $30(59)$ & $15(40)$ & $22(60)$ \\
\hline $\mathrm{Cz}$ & $133(21)$ & $502(79)$ & $127(50)$ & $127(50)$ & 0 & 0 & $64(51)$ & $62(49)$ & $22(44)$ & $29(56)$ & 0 & 0 \\
\hline Cn & $127(20)$ & $508(80)$ & $116(46)$ & $137(54)$ & 0 & 0 & $60(48)$ & $66(52)$ & $22(44)$ & $29(56)$ & 0 & 0 \\
\hline $\mathrm{Cxm}$ & $140(22)$ & $495(78)$ & $134(53)$ & $119(47)$ & 0 & 0 & $57(54)$ & $69(46)$ & $32(62)$ & $19(38)$ & 0 & 0 \\
\hline $\mathrm{Cfm}$ & $133(21)$ & $502(79)$ & $142(56)$ & $111(44)$ & 0 & 0 & $67(53)$ & $59(47)$ & $33(65)$ & $18(35)$ & 0 & 0 \\
\hline Ctr & $323(51)$ & $311(49)$ & $129(51)$ & $124(49)$ & $113(74)$ & $40(26)$ & $64(51)$ & $62(49)$ & $33(65)$ & $18(35)$ & $19(52)$ & $18(48)$ \\
\hline $\mathrm{Cpz}$ & $311(49)$ & $323(51)$ & $124(49)$ & $129(51)$ & $63(41)$ & $57(37)$ & $73(58)$ & $53(42)$ & $36(71)$ & $15(29)$ & $18(48)$ & $19(52)$ \\
\hline $\mathrm{Caz}$ & $331(52)$ & $305(48)$ & $127(50)$ & $126(50)$ & $63(41)$ & $90(59)$ & $68(54)$ & $38(46)$ & $29(56)$ & $22(44)$ & $19(52)$ & $18(48)$ \\
\hline $\mathrm{Ctx}$ & $305(48)$ & $331(52)$ & $132(52)$ & $121(48)$ & $96(63)$ & $57(37)$ & $64(51)$ & $62(49)$ & $29(56)$ & $22(44)$ & $18(48)$ & $19(52)$ \\
\hline $\mathrm{Clr}$ & $133(21)$ & $502(79)$ & $116(46)$ & $137(54)$ & $66(43)$ & $87(57)$ & $49(39)$ & $77(61)$ & $22(44)$ & $29(56)$ & $15(40)$ & $22(60)$ \\
\hline Do & $140(22)$ & $495(78)$ & $94(37)$ & $159(63)$ & $44(29)$ & $109(71)$ & $45(36)$ & $81(64)$ & $18(35)$ & $33(65)$ & $15(40)$ & $22(60)$ \\
\hline Cip & $292(46)$ & $343(54)$ & $91(63)$ & $162(37)$ & $130(85)$ & $23(15)$ & $40(32)$ & $86(68)$ & $29(56)$ & $22(44)$ & $30(80)$ & $7(20)$ \\
\hline Gem & $311(49)$ & $323(51)$ & $190(75)$ & $63(25)$ & $121(79)$ & $32(21)$ & $64(51)$ & $62(49)$ & $33(65)$ & $18(35)$ & $33(88)$ & $4(12)$ \\
\hline Of & $273(43)$ & $362(57)$ & $127(50)$ & $126(50)$ & $109(71)$ & $44(29)$ & $34(27)$ & $92(73)$ & $18(35)$ & $33(65)$ & $18(48)$ & $19(52)$ \\
\hline Cot & $133(21)$ & $502(79)$ & $119(47)$ & $134(53)$ & 0 & 0 & $35(28)$ & $91(72)$ & $19(38)$ & $32(62)$ & 0 & 0 \\
\hline Cfs & $311(49)$ & $323(51)$ & $124(49)$ & $129(51)$ & $90(59)$ & $63(41)$ & $66(52)$ & $60(51)$ & $18(35)$ & $33(65)$ & $16(44)$ & $21(56)$ \\
\hline Amc & $127(20)$ & $508(80)$ & $139(55)$ & $114(45)$ & $47(31)$ & $106(71)$ & $34(27)$ & $92(73)$ & $16(32)$ & $35(68)$ & $9(24)$ & $28(76)$ \\
\hline Pit & $406(64)$ & $229(36)$ & $134(53)$ & $119(47)$ & $89(58)$ & $64(42)$ & $72(57)$ & $54(43)$ & $22(44)$ & $29(56)$ & $33(88)$ & $4(12)$ \\
\hline Ipm & $635(100)$ & $0(0)$ & $253(100)$ & $0(0)$ & $152(100)$ & $0(0)$ & $126(100)$ & $0(0)$ & $51(100)$ & $0(0)$ & $37(100)$ & $0(0)$ \\
\hline
\end{tabular}

Figure 1 - Percentage of samples with bacterial growth

\section{Percentage of samples with Bacterial growth}

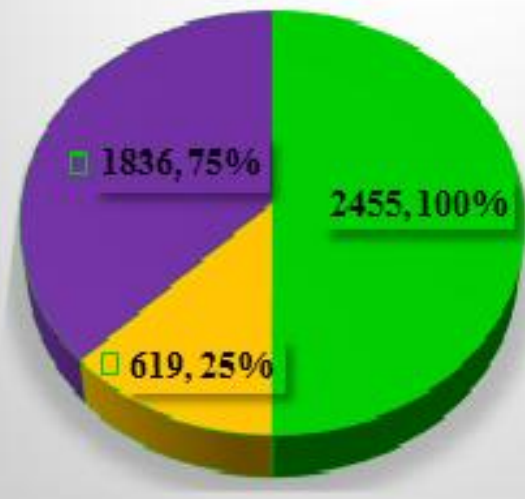

- TOTAL

$\square$ NO GROWTH

= GROWTH 
Table.4 Antibiotic susceptibility of gram positive bacteria to various groups of antibiotics

\begin{tabular}{|c|c|c|c|c|c|c|}
\hline \multirow{2}{*}{$\begin{array}{l}\text { Organisms } \\
\mathbf{n}(\%)\end{array}$} & \multicolumn{2}{|l|}{ S. aureus } & \multicolumn{2}{|l|}{ CONS } & \multicolumn{2}{|l|}{ Enterococci } \\
\hline & 334(18) & & 204(11) & & $43(2)$ & \\
\hline Antibiotics & Susceptible & Resistant & Susceptible & Resistant & Susceptible & Resistant \\
\hline Amp & $194(58)$ & $140(42)$ & $122(60)$ & $82(40)$ & $14(33)$ & $29(67)$ \\
\hline $\mathrm{Pi}$ & $197(59)$ & $137(41)$ & $120(59)$ & $84(41)$ & $29(67)$ & $14(33)$ \\
\hline $\mathrm{AK}$ & $197(59)$ & $91(41)$ & $139(68)$ & $53(32)$ & 0 & 0 \\
\hline Gen (HLG) & $160(48)$ & $115(52)$ & $114(56)$ & $90(44)$ & $43(100)$ & $0(0)$ \\
\hline $\mathrm{Cx}$ & $190(57)$ & $96(43)$ & $120(59)$ & $84(41)$ & 0 & 0 \\
\hline $\mathrm{Clr}$ & $73(22)$ & $173(78)$ & $39(19)$ & $165(81)$ & 0 & 0 \\
\hline Azm & $70(21)$ & $174(79)$ & $37(18)$ & \begin{tabular}{|l|}
$167(82)$ \\
\end{tabular} & 0 & 0 \\
\hline Do & $90(27)$ & $163(73)$ & $55(27)$ & 149(13) & $14(33)$ & $29(67)$ \\
\hline Cot & $67(20)$ & $178(80)$ & $65(32)$ & $139(68)$ & 0 & 0 \\
\hline Cip & $47(14)$ & $192(86)$ & $75(37)$ & $129(63)$ & $14(33)$ & $29(67)$ \\
\hline Gem & $43(13)$ & $194(87)$ & $96(43)$ & \begin{tabular}{|l|}
$108(57)$ \\
\end{tabular} & $29(67)$ & $14(33)$ \\
\hline Of & $43(13)$ & $195(87)$ & $63(31)$ & \begin{tabular}{|l|}
$141(69)$ \\
\end{tabular} & 14(33) & $29(67)$ \\
\hline $\mathrm{Cn}$ & $204(61)$ & $130(39)$ & $122(60)$ & $82(40)$ & 0 & 0 \\
\hline $\mathrm{Cxm}$ & $200(60)$ & $134(40)$ & $124(61)$ & $80(39)$ & 0 & 0 \\
\hline $\mathrm{Ctr}$ & $190(57)$ & $144(43)$ & $120(59)$ & $84(41)$ & 0 & 0 \\
\hline Cfs & $190(57)$ & $144(43)$ & $118(58)$ & $86(42)$ & 0 & 0 \\
\hline Amc & $197(59)$ & $137(41)$ & $120(59)$ & $84(41)$ & $29(67)$ & $14(33)$ \\
\hline Pit & $194(58)$ & $140(42)$ & $116(57)$ & $88(43)$ & $43(100)$ & $0(0)$ \\
\hline Van & $334(100)$ & $0(0)$ & $204(100)$ & 0 & $43(100)$ & $0(0)$ \\
\hline $\mathrm{Lz}$ & $334(100)$ & $0(0)$ & $204(100)$ & 0 & $43(100)$ & $0(0)$ \\
\hline
\end{tabular}

Figure 2 - Pus samples from $\mathrm{OP}$ and IP

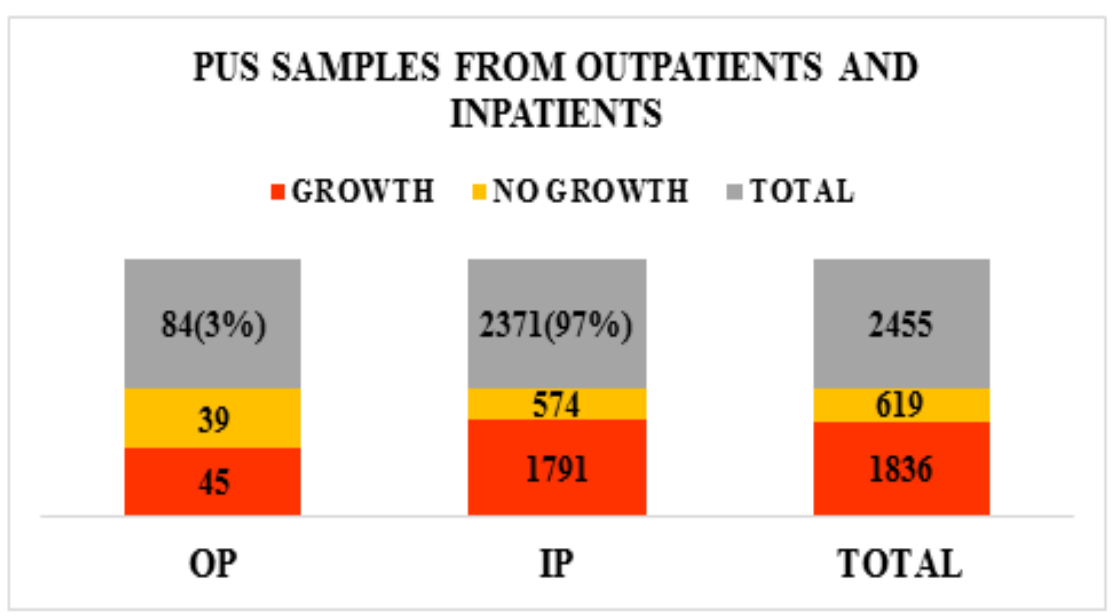

Gram positive bacteria were isolated from 581 (32\%) pus samples and gram negative bacteria were isolated from $1255(68 \%)$ pus samples. $73 \%$ males and $27 \%$ females showed bacterial growth. Out of the total samples bacteria was isolated from 84 (3\%) outpatients and 2371(97\%) inpatients from various wards of our hospital as in Figure 2 
Figure 3 - BACTERIAL ISOLATES FROM PUS SAMPLES

Total

CONS

204(11\%)

Enterococci $\quad 43(2 \%)$

Staph aureus

Acinetobacter $\square \mathbf{3 7}(\mathbf{2} \%)$

Citrobacter $\mathbf{5 1}(\mathbf{3} \%)$

Proteus sp

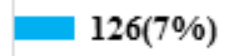

Pseudomonas

$153(8 \%)$

E.coli

253(14\%)

Klebsiella

$635(35 \%)$

Figure 4 - Gram negative bacilli producing ESBL in percentage

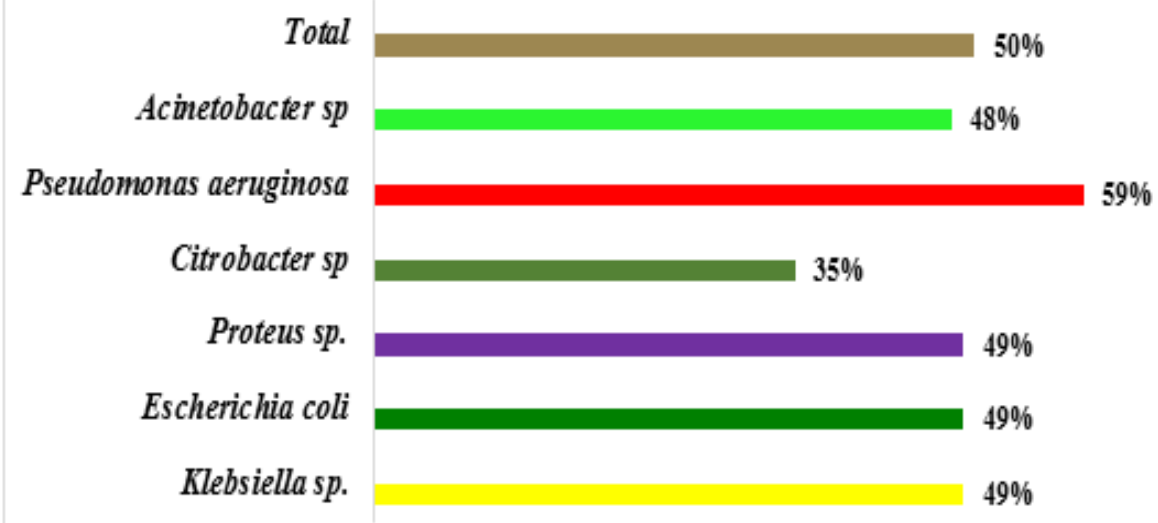

Figure.5 Phenotypic confirmatory double disc diffusion test for ESBL isolates

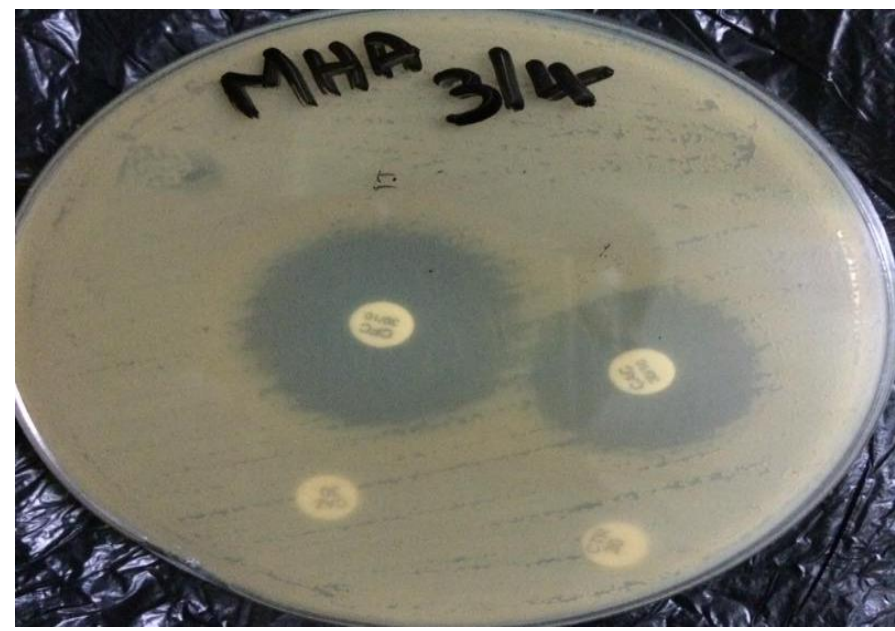


Figure 6

$$
\begin{gathered}
\text { Percentage of Antibiotic Susceptibility pattern of Klebsiella } \\
\text { species }
\end{gathered}
$$

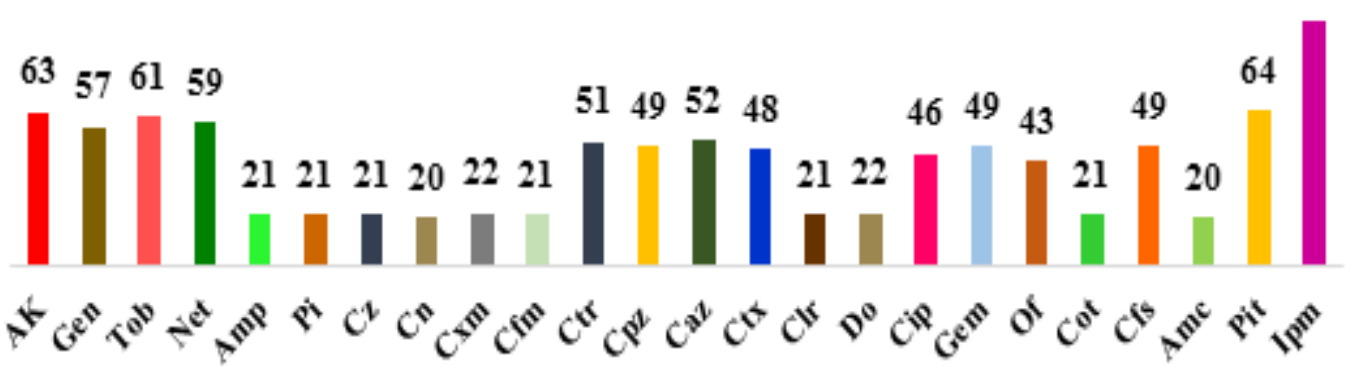

Figure 7-Percentage of Antibiotic susceptibility pattern of Escherichia coli

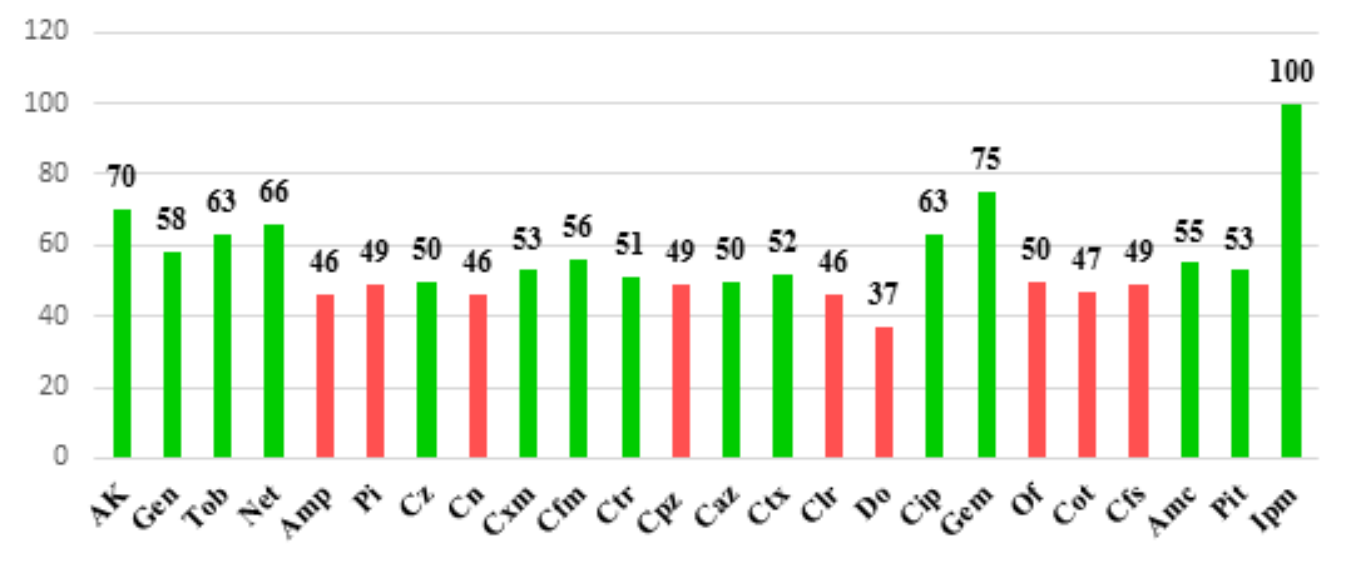

In the above figure all the E.coli isolates with sensitivity more than $50 \%$ is highlighted in green and those with $<=50 \%$ sensitivity is in red colour.

Figure 8 -Percentage of antibiotic susceptibility pattern of Pseudomonas aeruginosa Susceptible $\square$ Resistant

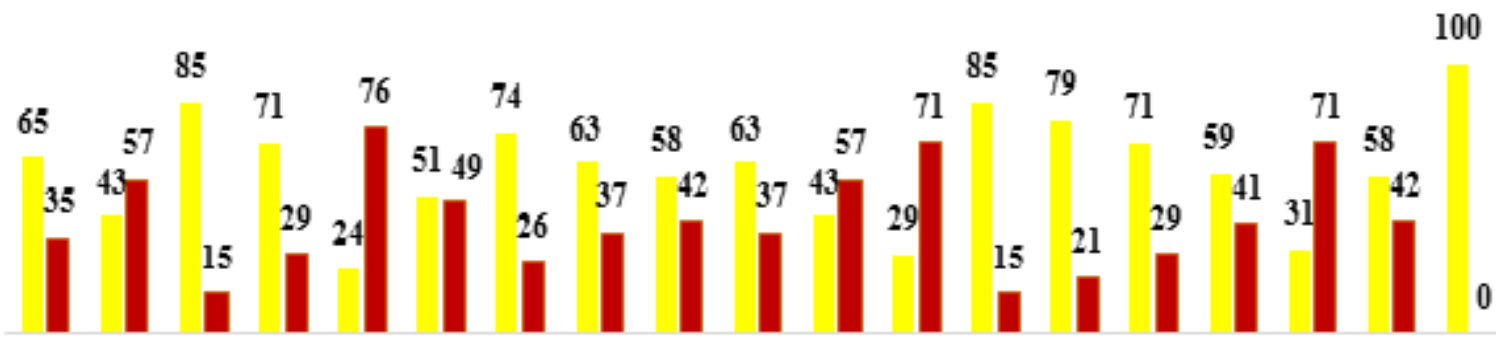

AK Gen Tob Net Amp Pi Ctr Cpz Caz Ctx Clr Do Cip Gem Of Cfs Amc Pit Ipm 
As most of the empirically preferred antibiotics are almost susceptible to gram negative bacilli the use of the same as above shall be implemented for treatment of Klebsiella infections. This finding is similar to correlate with Zhang et al., (2014) who reported predominance of $K$. pneumoniae, $E$. coli, S. aureus and $P$. aeruginosa in pus samples from patients with severe wound infection.

E. coli isolates exhibited antibiotic susceptibility of $100 \%$ to imipenem, followed by $75 \%$ to gemifloxacin, $70 \%$ to amikacin, $63 \%$ to tobramycin, $63 \%$ to ciprofloxacin and $49 \%$ sensitivity to cefaperazonesulbactum combination.

Pseudomonas aeruginosa is $100 \%$ sensitive to Imipenem, $85 \%$ to tobramycin, $85 \%$ to Ciprofloxacin, $71 \%$ to netilmicin, followed by Piperacillin (51\%), Ceftazidime (58\%), cefaperazonesulbactum (59\%), and piperacillintazobactum (58\%).

These studies were compared to other studies like Soumya Kaup and Jaya Sankarankutty (2014), Rajeshwaretal (2014) as these showed similarities in antibiotic sensitivity pattern.

Gram positive bacteria mainly $S$. aureus exhibited $100 \%$ sensitivity to vancomycin and linezolid followed by $70 \%$ sensitivity to macrolide group of antibiotics, $60 \%$ sensitivity to all beta lactam antibiotics and $59 \%$ to aminoglycosides.

Multidrug resistance (Resistant to one or more antibiotic classes) was found to be predominant in Klebsiella pneumonia followed by Staphylococcus aureus. Hence indiscriminate use of antibiotics and irregular treatment shall be avoided as this study has expressed the percentage of bacterial isolates susceptible to all antibiotics of common use. The spread of ESBL-producing bacteria has been strikingly rapid worldwide, indicating that continuous monitoring systems and effective infection control measures are absolutely required as per the study by Basavaraj C. Metri et al., (2011).

The cefoxitin disc diffusion method, as recommended by the Clinical and Laboratory Standards Institute, was found to be a reliable method for MRSA detection Priya Datta et al., (2011). Incidence of 35\%- 40\% MRSA was detected in study by Raghabendra Adhikari et al., (2017) and Rashmi et al., (2017). Mohammad Fareed Khan et al., (2015) also has detected that $35 \%$ of all S. aureus infections had been caused by MRSA.

In this study ESBL isolates has been detected by different gram negative bacilli with high incidence in Klebsiella $\mathrm{sp}$ followed by Escherichia coli, Proteus sp, Citrobacter species among gram negative bacilli under family Enterobacteriaeceae. Among nonfermenting gram negative bacilli high incidence of ESBL was detected in Pseudomonas aruginosa followed by Acinetobacter species.

All MRSA isolates were sensitive to vancomycin, linezolid, mupirocin and rifampicin similar to the study by Arunava Kali et al., (2013). Verma et al., has reported a rapid increase in MRSA prevalence, from $12 \%$ to $80.89 \%$, over seven years, in a tertiary care centre at Indore. As per study by Srikanth et al., (2013), 40\% among Staphylococcus species were methicillin resistant. This is in similarity to our study.

In contrast, very low incidence of $26 \%$ and high incidence of $78 \%$ had been reported by Pandey et al., (2012) and Masoumeh Navidinia et al., (2015).

This variation may be due to other associated factors like less adherence to hygienic 
practices, sample load of patients where more possibility for transmission of MRSA shall be considered to be the main cause.

ESBL production was detected in all the gram negative bacilli in varying percentage. Total gram negative bacilli producing ESBL was $626(50 \%)$. Highest incidence was due to ESBL isolates of Pseudomonas aeruginosa 90 (59\%) followed by Klebsiella sp 312(49\%), E. coli 124 (49\%), Proteus sp. 62(49\%), Acinetobacter sp 18 (48\%) and Citrobacter sp $18(35 \%)$.

Klebsiella pneumoniae was the most common ESBL producing Enterobacteriaceae, followed by $E$. coli and others, as per Basavaraj C. Metri et al., (2011) which is similar to this study. Nazneen Siddiqui et al., 2014 has reported $61 \%$ were found to be ESBL positive by the CDDT method. The different gram negative bacilli identified were Klebsiella pneumoniae (47\%). This is also similar to figures reported in our study.

ESBL production was most common in Klebsiella pneumoniae (74\%), followed by $E$. coli (62\%) as per Ankur Goyal et al., (2009). This was found to be higher than that reported in our study. Imipenem showed the highest rate of sensitivity (97\%) almost similar to our study with $100 \%$ sensitivity to imipenem.

Baby Padmini et al., have shown $40 \%$ and $41 \%$ ESBL positivity among $K$. pneumoniae and $E$. coli respectively and Vinod Kumar et al., from Gulbarga reported $16.8 \%$ and $48.6 \%$ of $E$. coli and $K$. pneumoniae respectively as the ESBL producers. This is similar to finding in our study. Basavaraj et al., (2011).

As per Ankur Goyal et al., (2009), occurrence of ESBL was reported to be $63.6 \%$ in $E$. coli and $66.7 \%$ in $K$. pneumoniae. This figure is higher than that reported in our study which may be due to variable distribution of normal microbial flora and indiscriminate use of antibiotics leading to increasing multi drug resistance.

As per the study by Shaoyi Zhang et al., (2014) majority of patients admitted with wound infections were due to trauma or injury and among those who were admitted to the emergency room immediately following trauma, majority of cases were complicated with shock. High degree of resistance was observed among gram negative organisms to beta lactam groups of antibiotics. The percentage of oxacillin resistance among staphylococcal isolates was $33.1 \%$, but all were sensitive to vancomycin as per the study by Iff at Javeed et al., (2011) and these correlate with our findings in this study.

No bacterial isolates was found to be sensitive to all antibiotics tested. Isolated bacteria showed multi drug resistance to the commonly used antibiotics in the hospital.

Most of the Gram negative bacteria and Gram positive isolates were sensitive to Gentamicin and Vancomycin, respectively as per the study by Mengesh et al., (2014) which is similar to this study

As most of the figures in various studies conducted since years were similar to the findings in our study the rate of incidence of bacterial isolates with their antibiotic susceptibility pattern, sociodemographic factors related to wound infections have been widely described.

Similarly, all the relevant information about the bacterial pathogens isolated from the pus samples and antibiotic guideline to be followed by all the clinicians for empirical treatment of the patients in case of accidental trauma, other emergencies and for outpatients has been outlined in this study. 
This would be the baseline for formulation of policies for prevention of emerging multi drug resistant bacterial isolates in our hospital. In addition to this proper hygiene and sanitation measures shall be adopted by all the health care personnel of our hospital among which adoption of hand hygiene which would be considered to be of prime importance.

Most important points to be considered includes negligence on patients part, incomplete treatment schedules, antibiotic misuse, self-prescription, misprescription, lack of regional antibiogram data, and limited knowledge about multidrug-resistant isolates and antimicrobial resistance among clinicians.

Routine review and updates of antimicrobial susceptibility profiles of clinical isolates will help in formulating the most appropriate antibiotic to be prescribed in our hospital, dose-regimen and treatment schedule against wound infections but also help in decreasing the emerging and alarming drug resistant bacterial isolates.

All the patients received in our hospital has to be made aware of the need for hygiene and sanitation measures which shall be an insight to the society for prevention of emerging multidrug resistant isolates and spread of the same.

In conclusion, wound infections are more prevalent due to lack of awareness about hygienic practices, empirical treatment of patients with irrelevant antibiotics use.

This study was carried out in our facility to provide relevant information with regard to bacteria causing wound infections.

Antibiotic susceptibility to various group of antibiotics were interpreted based on CLSI guidelines which has outlined the basic idea for antibiotics that shall be prescribed in our facility empirically.

As emerging antibiotic resistance of bacterial isolates has posed a threat to the entire society this study has enlightened the necessity of proper selection of antibiotics and to administer the appropriate dosage and duration of their use as per the pharmacological protocols. Hence strict adherence to formulated antibiotic policies and infection control measures suitable for our set up has to be considered essential.

This study has also defined the socio demographic factors in epidemiology of wound infections. Incidence of wound infections and multidrug resistant organisms has also been derived. As this type of prospective study has not been conducted so far in our hospital this data would be helpful to cater the needs of the patients with wound infections.

\section{References}

Ananth and S.Rajan. Isolation and Screening of Pathogenic Bacteria from Wound Infections. International Journal of Current Pharmaceutical Research.2014; 6(3): 15

AnkurGoyal,K.N. Prasad, Amit Prasad, Sapna Gupta, Ujjala Ghoshal and Archana Ayyagari, Extended spectrum $\beta$ lactamases in Escherichia coli Escherichia coli and Klebsiella pneumoniae and associated risk factors,Indian J Med Res 129, June 2009, pages 695-700)

Akimoto Y et al., Mochizuki Y, Uma A, Omata $\mathrm{H}$, Shibutani J, Nishimura $\mathrm{H}$, et al., Amoxicillin concentration in pus from abscess caused by odontogenic infection. Gen Pharmacol 1994; 25:111-13. 
Aniruddha S Mundhada, SunitaTenpe,,A study of organisms causing surgical site infections and their antimicrobial susceptibility in a tertiary care Government Hospital, IJPM, Year: 2015 | Volume: 58 | Issue: 2 | Page: 195-ᄀר200

Ashby E, Haddad FS, O'Donnell E, Wilson AP (2010). How will surgical site infection be measured to ensure "high quality care for all"? J Bone Joint Surg Br 92: 1294-1299.

Aizza Zafar, Naeema Anwar and Hasanejaz. Bacteriology of infected wounds - A study Conducted at Children's Hospital Lahore. Biomedica.2008; 24:71-74.

ArunavaKali, Selvaraj Stephen, SivaramanUmadevi, Shailesh Kumar, NoyalMariyaJoseph,

SreenivasanSrirangaraj, Changing Trends in Resistance Pattern of Methicillin Resistant Staphylococcus aureus, Journal of Clinical and Diagnostic Research. 2013 Sept, Vol7(9): 1979-1982

VG Bhat and SD Vasaikar. Bacteriological profile and antibiogram of aerobic burn wound isolates in Mthatha, Eastern Cape, South Africa. South Africa journal of Epidemiol Infect 2010; 25(4):16-19.

Baby Padmini S, Appalaraju B. Extended spectrum $\quad \beta$-lactamasesin urinary isolates of Escherichia coli and Klebsiellapneumoniae-Prevalence and susceptibility pattern in a tertiary care hospital. IndianJ Med Microbiol2004; 22(3):172-174.

Basavaraj C. Metri et al., ESBL among Enterobacteriaceae, and Journal of Clinical and Diagnostic Research, 2011 June, Vol-5(3): 470-475

Bilal Ahmad Mir, Dr. SRIKANTH, Prevalence and antimicrobial susceptibility of methicillin resistant Staphylococcus aureus and Coagulase- negative staphylococci in a tertiary care hospital,Asian J Pharm Clin Res, Vol 6, Suppl 3, 2013, 231-234

P. G. Bowler, B. I. Duerden, and D. G. Armstrong, "Wound microbiology and associated approaches to wound management,"Clinical Microbiology Reviews, vol.14, no.2, pp.244-269, 2001

Clinical Laboratory Standards Institute Performance Standards for antimicrobial susceptibility testing $\mathrm{M}$ 100, 27 th edition, January 2017

E. Cerceo, S. B. Deitelzweig, B. M. Sherman, and A. N. Amin,"Multidrug-resistant gram-negative bacterial infections in the hospital setting: overview, implications for clinical practice, and emerging treatment options," Microbial Drug Resistance, vol. 22, no. 5, pp. 412-431, 2016.

BN Chaudhuri et al.,2007) C Rodrigues, V Balaji, R Iyer, Incidence of ESBL Producers amongst Gram-negative Bacilli Isolated from Intra-abdominal Infections across India (Based on SMART Study, 2007 Data),JAPI, May 2011, Vol.59,6 pages)

A.L.Cogen, V.Nizet, and R.L.Gallo,Skin micro biota: a source of disease or defence ?" British Journal of Dermatology, vol. 158, no.3, pp.442455, 2008.

DagnachewMuluye, YitayihWondimeneh, GetachewFerede, TesfayeNega, KasawAdane, BeleteBiadgo, Bacterial isolates and their antibiotic susceptibility patterns among patients with pus and/or wound discharge at Gondar university hospital, BMC Research Notes 2014, 7:619

Dryden,M.S. "Complicated skin and soft tissue infection," Journal of Antimicrobial Chemotherapy, vol. 65, supplement 3, pp.iii35-iii44,2010. 
Ehmer Al-Ibran, Sidra Meraj, MahwishNasim, Maria Khan2 and Masood Hussain Rao. Pattern of Microorganisms Isolated from Flame Burn Wounds, and Their Trends of Susceptibility to Antibiotic During Past Three Years. Journal of the Dow University of Health Sciences Karachi. 2013; 7: 49-53.

Ezekiel OlugbengaAkinkunmi, Abdul-Rashid Adesunkanmi, Adebayo Lamikanra, Pattern of pathogens from surgical wound infections in a Nigerian hospital and their antimicrobial susceptibility profiles, African Health Sciences, Vol 14, Issue 4, December 2014;802-809

Farah Siddiqi, Madahihah-bint-E-Masood, Noor-Us-Saba, AbdusSamad, MazharQayyum and Ali Abbas Qazilbash., Antibiogram Sensitivity Pattern of Methicilline Resistant S. aureus isolates from Pus Sample., Pakistan Journal of Biological Science. 2002; 5(4): 491-493.

FarzanaR,Shamsuzzaman SM, Mamun KZ, and Shears P. Antimicrobial susceptibility pattern of extended spectrum beta-lactamase producing gram-negative bacteria isolated from wound and urine in a tertiary care hospital, Dhaka City, Bangladesh. Southeast Asian J Trop Med Public Health. 2013; 44(1): 96-103.

EA George, S Sankar, MV Jesudasan, C Sudandiradoss, B NandagopalIncidence of extended spectrum beta lactamase producing Escherichia coli among patients, healthy individuals and in the environment,Year: 2014, Volume: 32, Issue: 2, Pages: 172-174)

HimaBinduMantravadi, Mallikarjuna Reddy Chinthaparthi, Shravani V, Aerobic isolates in pus and their antibiotic sensitivity pattern: a study conducted in a teaching hospital in Andhra Pradesh, International Journal of Medical
Science and Public Health | 2015 | Vol 4 Issue 8

Haitham M. Al-Habib, Asmaa Z. Al-Gerir, Ansam M. Hamdoon. Profile of Pseudomonas aeruginosa in burn infection and their antibiogram study. Annals of the College of Medicine. 2011; 37:1-2.

Iman A. Hussien Khalid A. Habib Kifah A. Jassim. Bacterial Colonization of Burn wounds. J. Baghdad for Science.2012; 9(4):623-631.

IffatJaveed, RbeenaHafeez and M. Saeed Anwar, Antibiotic susceptibility pattern of bacterial isolates from patients admitted to a tertiary care hospital in Lahore Biomedica vol. 27 (Jan. - Jun. 2011) d:Liomedica vol.27, Jan. - Jun. 2011 bio-18.doc p. 19 - 23

J.Iredell, J.Brown, andK.Tagg, "AntibioticresistanceinEnterobacteriace ae: mechanisms and clinical implications," British MedicalJournal, vol.352, ArticleIDh6420, 2016.

KrituPanta, Prakash Ghimire, Shiba Kumar Rai, ReenaKiranMukhiya, Ram Nath Singh, Ganesh Rai. Antibiogram Typing of Gram Negative Isolates in Different Clinical Samples of a Tertiary Hospital. Asian Journal of Pharmaceutical and Clinical Research.2013; 6:153-156.

A.R. Kumar. Antimicrobial sensitivity pattern of Klebsiella pneumonia isolated from pus from tertiary care hospital and issues related to the rational selection of antimicrobials. Journal of chemical and pharmaceutical research, 2013; 5 (11): 326-331.

Koneman'scolor atlas and textbook of diagnostic microbiology, 6th ed ( $p p$ 624-62). Philadelphia, PA: Lippincott Williams and Wilkins, 2006b.

Kelwin W.S. (1999). Anti-microbial therapy for diabetic foot infections, Post Grad. Med., 106: 22-28 
Lamikanra, Shinagawa N, Hirata K, Katsuramai T. et al.,, Bacteria isolated from surgical infections and its susceptibilities to antimicrobial agents special references to bacteria isolated between April 2003 and March 2004. Japan Journal of Antibiotics 2005; 58(2): $123-158$.

Lilani SP, Jangale N, Chowdhary A, Daver GB. Surgical site infection in clean and clean- contaminated cases. Indian Journal of Medical Microbiology 2005; 23:249-25211.

Lucinda J Bessa1, Paolo Fazii2, Mara Di Giulio3 and Luigina Cellini3 Bacterial isolates from infected wounds and their antibiotic susceptibility pattern: some remarks about wound infection, International Wound Journal ISSN 1742-4801, International Wound Journal (C) 2013 Blackwell Publishing Ltd and Medicalhelplines.com Incdoi: 10.1111/iwj. 12049

Maria Ayub, HumaRizwan, Safina Siddique and Ushna Maryam, Isolation of Pathogens Causing Sepsis, Pus and Infected Wounds from Critical Care Unit: A Retrospective Study, Annals of Clinical and Laboratory Research ISSN 2386-5180, 2015, Vol. 3, No. 4: 50

Mangram AJ, Horan TC, Pearson ML, Silver LC, Jarvis WR. Guideline for prevention of surgical site infection, 1999. Hospital Infection Control Practices Advisory Committee. Infection Control Hospital Epidemiology 1999; 20(4):250-278.

Mengesha et al., Aerobic bacteria in postsurgical wound infections and pattern of their antimicrobial susceptibility in Ayder Teaching and Referral Hospital, BMC Research Notes 2,7:575, http://www.biomedcentral.com/17560500/7/575

Mohammad Fareed Khan, S.K.B. Patil, Farah Aziz Khan., Prevalence and Drug
Resistance Pattern of Staphylococcus aureusClinical Isolates in Bastar Region, Journal of Clinical and Analytical Medicine2015;6(5): 628-32

Mathur T, Singhal S, Khan S, Upadhyay DJ, Fatma T, Rattan A. Detection of biofilm formation among the clinical isolates of Staphylococci:an evaluation of three different screening methods. Indian $\mathbf{J}$ Med Microbiol 2006; 24(1):25-9

Mulu A, Moges F, Tesema B, Kassu A: Pattern and multiple drug resistance of bacterial pathogens isolated from wound infection at University of Gondar teaching hospital, North west Ethiopia. Ethiop Medi J 2006, 44:125131.

Navidinia, FatemehFallah, BehnazLajevardi, Mehdi Shirdoost, JamshidJamali, Epidemiology of Methicillin-Resistant Staphylococcus aureusIsolated From Health Care Providers in Mofid Children Hospital, Arch Pediatr Infect Dis. 2015;3(2):e16458 DOI: 10.5812/pedinfect. 16458

Nichols RL. Current Strategies for Prevention of Surgical Site Infections. Curr Infect Dis Rep 2004; 6(6):426434.

Oluwatosin OM. Surgical Wound Infection: A General Overview. Annals of Ibadan Postgraduate Medicine 2005; 3(2):2631

Pandey S., Raza M. S., Bhatta C. P Prevalence and Antibiotic Sensitivity Pattern of Methicillin-ResistantStaphylococcus aureus in Kathmandu Medical College Teaching Hospital, Journal of Institute of Medicine, April, 2012;34:1,13-17

PriyaDatta, Neelam Gulati, NidhiSingla, Hena Rani Vasdeva, KiranBala, JagdishChander and VarshaGupta,Evaluation of various methods for the detection of meticillinresistant Staphylococcus aureus strains and susceptibility patterns, Journal of 
Medical Microbiology (2011), 60, 1613-1616

S. Rajeshwar Rao, L. Jaya Lakshmi1, S.Pavani, VijendraKawle and S. Jaya Prakash. Bacteriological Profile, Antibiogram of Burn wound Isolates and Detection of MRSA and ESBL Production at Tertiary Care Hospital, Hyderabad. World Journal of Pharmacy and Pharmaceuticals Sciences. 2014; 3:1691-1698.

S. Rashmi, Krishna and SumairaQayoom,Prevalence of MRSA among Clinical Isolates of Staphylococcus aureusand its Antibiotic Susceptibility Pattern at a Tertiary Care Hospital, International Journal of Current Microbiology and Applied Sciences,ISSN: 2319-7706 Volume 6 Number 1 (2017) pp. 747-749 http://dx.doi.org/10.20546/ijcmas.2017. 601.088

RaghabendraAdhikari, Narayan Dutt Pant, SanjeevNeupane, Detection of Methicillin Resistant Staphylococcus aureus and Determination of Minimum Inhibitory Concentration of Vancomycin for Staphylococcus aureus Isolated from Pus/Wound Swab Samples of the Patients Attending a Tertiary Care Hospital in Kathmandu, Nepal, Canadian Journal of Infectious Diseases and Medical Microbiology,Volume 2017, Article ID 2191532, 6 pages https://doi.org/10.1155/2017/2191532

RugiraTrojan,LovelyRazdan,andNasib Singh, Antibiotic Susceptibility Patterns of Bacterial Isolates from Pus Samples in a Tertiary Care Hospital of Punjab, India International Journal of Microbiology,Volume 2016, Article ID 9302692, 4 pages

K.N.Ravichitra, P.Hema Prakash, S. Subbarayudu and U. Sreenivasa Rao. Isolation and antibiotic sensitivity of
Klebsiella pneumonia from pus, sputum and urine samples. International journal of current microbiology and applied science. 2014; 3(3): 115-119.

Sanders CC, Barry AL, Washington JA, Shubert C, Moland ES, Traczewski MM, Knapp C, Mulder R. Detection of ESBL producing members of the family Enterobacteriaceae with the Vitek ESBL test. J ClinMicrobiol 1996; 34: 2997-3001)

Dr.R.Sarathbabu, $\quad$ Dr.T.V.Ramani, Dr.K.Bhaskararao and Dr.Supriya Panda. Antibiotic susceptibility pattern of Klebsiellapneumoniae isolated from sputum, urine and pus samples. IOSR Journal of Pharmacy and Biological Sciences.2012; 1:4-9.

S. Suganya., R. Bharathidasan, G.Senthilkumar, P.Madhanraj and A. Panneerselvam. Journal of Chemical and Pharmaceutical Research. 2012; 4(3):1846-1850.

SoumyaKaup and Jaya Sankarankutty. Prevalence and antimicrobial susceptibility patterns of bacteria isolated from skin and wound infections. Journal of Microbiology and Biotechnology Research.2014; 4(2): 3945.

A.Scalise, A. Bianchi, C.Tartaglioneetal, "Micro environment and microbiology of skin wounds: the role of bacterial biofilms and related factors," Seminars in Vascular Surgery, vol. 28, no. 3-4, pp.151-159, 2015.

Subrata Roy, Bacteriological Profile of PostOperative Wound Infection, Asian Journal of Biomedical and Pharmaceutical Sciences, 6(53), 2016, 44-46.)

Shaoyizhang, Leleren, Yousheng Li, Jian Wang, Wenkui Yu, Ning Li and Jieshou $\mathrm{Li}$, Bacteriology and drug susceptibility analysis of pus from patients with severe intra-abdominal infection 
induced by abdominal trauma,Experimental and therapeutic Medicine 7: 1427-1431, 2014

TsegayeSewunet, YohanesDemissie, AdaneMihret, TamratAbebe, Bacterial profile and antimicrobial susceptibility pattern of isolates among burn patients at Yekatit hospital burn center, Addis,Ababa, Ethiopia,Ethiop J Health Sci. Vol. 23, No. 3 November 2013

Verma S, Joshi S, Chitnis V, Hemwani N, Chitnis D. Growing problem of methicillin resistant staphylococci Indian scenario. Indian J Med Sci. 2000; 54:535-40.

Vinodkumar CS, Neelagund YF. Extendedspectrum $\quad \beta$-lactamase mediated resistance to third generation cephalosporins among Klebsiellapneumoniae in neonatal septicemia. J Indian Paediatr; 2004: 41: 97-99

Vijeta Sharma, GeetaParihar, Vijaylaxmi Sharma, Harshita Sharma, A Study of Various Isolates from Pus Sample with Their Antibiogram from Jln Hospital, Ajmer, Journal of Dental and Medical Sciences (IOSR-JDMS) e-ISSN: 22790853, p-ISSN: 2279-0861. Volume 14, Issue 10 Ver. VI (Oct. 2015), PP 64-68 www.iosrjournals.org DOI: $10.9790 / 0853-141066468$ www.iosrjournals.org 64 | Page.

\section{How to cite this article:}

Sukumar Nirmala and Rajesh Sengodan. 2017. Aerobic Bacterial Isolates and their Antibiotic Susceptibility Pattern from Pus Samples in a Tertiary Care Government Hospital in Tamilnadu, India. Int.J.Curr.Microbiol.App.Sci. 6(6): 423-442. doi: https://doi.org/10.20546/ijcmas.2017.606.050 